\title{
MAP-based Timing Recovery for Magnetic Recording
}

\author{
Raman Venkataramani \\ Seagate Technology \\ 1251 Waterfront Place, Pittsburgh, PA 15222 \\ Email: ramanv@ieee.org
}

\author{
M. Fatih Erden \\ Seagate Technology \\ 1251 Waterfront Place, Pittsburgh, PA 15222 \\ Email: fatih.erden@seagate.com
}

\begin{abstract}
We consider the problem of timing recovery in magnetic recording channels based on MAP estimation of the timing information. The read and write clocks are modeled as random walk processes that allow for slowly varying phase and frequency offsets of the clocks. We propose a new timing error detector (TED) that provides sufficient statistics about the instantaneous timing error. Using the clock models and the new TED, the MAP estimates of the sampling times are derived. This method is shown to be more robust than the conventional algorithm based on the Mueller and Müller TED and easily implementable for a small additional complexity.
\end{abstract}

\section{INTRODUCTION}

Timing recovery, also known as clock synchronization, is an important front-end operation in many communication systems. Magnetic recording is one such system where we identify the write-head as the transmitter and the read-head as the receiver. The desire to push recording densities higher has caused today's recording devices to operate at high recording densities and low signal-to-noise ratio (SNR) making the problem of timing recovery more challenging.

Traditional timing recovery architectures consist of a timing error detector (TED) whose function is to process the received samples to produce a quantity that is a measure of the timing phase error. This is further passed through a loop filter to produce the correction to the timing phase, which in turn drives the sampler through a voltage controlled oscillator (VCO). The system is decision-directed, i.e., the detected bits are used by the timing recovery algorithm with the assumption that they are error free. The conventional timing recovery system is illustrated in Fig. 1. A commonly used TED is the Mueller and Müller (MM) TED [1] which is based on heuristic arguments. The MM method performs quite well at high SNRs where the

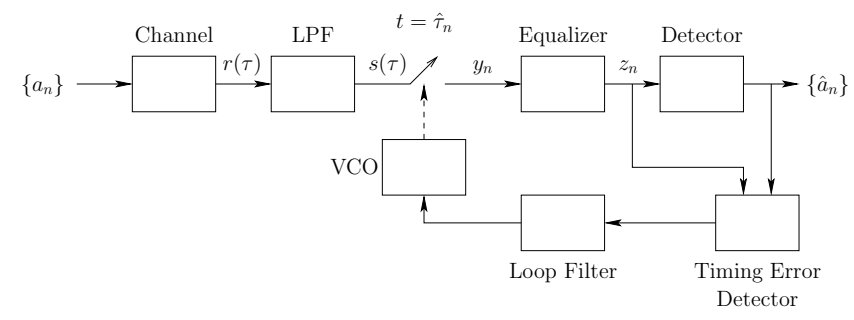

Fig. 1. Conventional timing recovery.

cycle slip rates [2] are generally very low. The resulting bit error rates are nearly equal to that of the perfect timing case. However, at lower SNRs, the system is plagued by cycle slips and the bit error rate rises. A thorough discussion of various timing recovery algorithms can be found in [3].

The purpose of this work is to revisit the problem of timing recovery with an attempt to improve the system performance. Our approach is to combine all the functional blocks of the timing recovery system, viz. the TED and the loop filter into one block and optimize it in a unified way. We derive the TED that provides sufficient statistics on the instantaneous timing error. Combining this with random walk models for the read and write clocks we derive the maximum a posteriori (MAP) and minimum-mean square error (MMSE) estimates of the timing information, akin to Kalman filtering. Using simulations, we show that the new method is robust and tracks changes in the clock phase and frequency extremely well.

\section{Signal And Channel Models}

We model the readback waveform from a magnetic recording medium as follows

$$
r(\tau)=\sum_{m} b_{m} h_{T}(f(\tau)-m T)+z(\tau)
$$

where $b_{m}=\left(a_{m}-a_{m-1}\right) / 2$ is the transition sequence corresponding to the written bit sequence $\left\{a_{m}= \pm 1\right\}$ which represents the magnetization of the medium, $T$ is the bit-width, $h_{T}(t)$ is the transition response of the system defined as the response to a transition of the magnetization from $-1 \rightarrow+1$, $z(t)$ is the additive Gaussian electronic noise with power spectral density of height $N_{0} / 2$, and $f(\tau)$ is a continuous, monotonically increasing function that maps the time $\tau$ on the read-clock to the time $\tau^{\prime}=f(\tau)$ on the write-clock. Clock imperfections cause $f(\tau)$ to be different from its ideal form: $f(\tau)=\tau$. We assume that the input bits are independent and identically distributed (IID). For example, for perpendicular recording, the transition response is

$$
h_{T}(t)=\operatorname{erf}\left(\frac{2 t \sqrt{\ln 2}}{W}\right)
$$

where $\operatorname{erf}(x)=\frac{2}{\sqrt{\pi}} \int_{0}^{x} e^{-t^{2}} d t$ is the error function and $W$ is the pulse width defined by the limits $t= \pm W / 2$ where the derivative of the transition response is one half of its peak value. The model (1) includes the effects of imperfections in 
the read and write clocks. The fundamental problem of timing recovery is to use the readback waveform to estimate the writeclock, i.e., to determine where to sample the waveform.

At moderate normalized densities (as in today's recording systems) the channel response $h_{T}(t)$ is bandlimited to the frequencies $\pm 1 / 2 T$ for practical purposes. Furthermore, since the slope of $f(\tau)$ is close to unity, we may approximate $h_{T}(f(t))$ as being bandlimited to $\pm 1 / 2 T$. Thus, passing the readback signal $r(\tau)$ through an analog low-pass filter with cutoff frequencies at $\pm 1 / 2 T$ approximately produces

$$
s(\tau)=\sum_{m} b_{m} h_{T}(f(\tau)-m T)+w(\tau) .
$$

The term $w(\tau)$ is the additive Gaussian noise bandlimited to $[-1 / 2 T, 1 / 2 T]$ with a flat spectrum of $N_{0} / 2 T$. From (3) it is clear that the ideal (desired) sampling locations with respect to the read-clock are $\tau_{m}:=f^{-1}(m T)$. We adopt a discrete-time second order random walk model for $\tau_{m}$ :

$$
\begin{aligned}
& \lambda_{n}=A \lambda_{n-1}+v_{n} \\
& \lambda_{n}=\left(\begin{array}{c}
\tau_{n} \\
\mu_{n}
\end{array}\right), \quad v_{n}=\left(\begin{array}{l}
\xi_{n} \\
\zeta_{n}
\end{array}\right), \quad A=\left(\begin{array}{ll}
1 & T \\
0 & 1
\end{array}\right) .
\end{aligned}
$$

Here $\mu_{n} \simeq 1$ represents the local frequency of the write-clock relative to the read-clock, while $\xi_{n}$ and $\zeta_{n}$ are IID Gaussian random walk steps with $\mathrm{E}\left[v_{n} v_{m}^{T}\right]=K_{v}(T) \delta_{m n}$. Denote the covariance of $\left(\lambda_{n}-A^{n} \lambda_{0}\right)$ by $K_{v}(n T)$. Then, for consistency we must satisfy

$$
K_{v}(n T)=A K_{v}(n T-T) A^{T}+K_{v}(T)
$$

because the two sides of (5) are simply two different ways of expressing the covariance of

$$
\begin{aligned}
\lambda_{n}-A^{n} \lambda_{0} & =v_{n}+A \lambda_{n-1}-A^{n} \lambda_{0} \\
& =v_{n}+A\left(\lambda_{n-1}-A^{n-1} \lambda_{0}\right) .
\end{aligned}
$$

It is an easy exercise to prove that $K_{v}(\tau)$, extended to $\tau \in$ $\mathbb{R}$, must be of the form

$$
K_{v}(\tau)=\left(\begin{array}{cc}
\sigma_{R}^{2} \tau+\sigma_{S}^{2} \tau^{3} / 3 & \sigma_{S}^{2} \tau^{2} / 2 \\
\sigma_{S}^{2} \tau^{2} / 2 & \sigma_{S}^{2} \tau
\end{array}\right)
$$

where the off-diagonal terms above are specifically chosen to satisfy the consistency condition (5) for any index $\tau=n T$. We could also have derived (6) directly from a second order continuous random walk model for $\tau(t)=f^{-1}(t)$.

We denote $K_{v}(T)$ simply by $K_{v}$. The parameters $\sigma_{R}$ and $\sigma_{S}$ control the rates of clock phase and frequency drift. We point out that the second order random walk itself is not new in the literature [4], [5]. However, $K_{v}$ was always chosen to be diagonal, ignoring the consistency condition.

\section{DERIVATION OF Optimal SYNCHRONIZER}

Fig. 2 shows the proposed timing recovery architecture with a single block labeled "Timing Estimator" replacing the TED, loop filter and the voltage controlled oscillator (VCO). We use a decision-directed approach assuming that $a_{n}=\hat{a}_{n}$ most of the time. A preamble field is used at the start of each sector to ensure proper acquisition of the timing information. Note that it is better to process the actual samples $y_{n}$ rather than of the equalized sequence $z_{n}$ (as in Fig. 1) owing to the data processing theorem [6]. In this section, we derive the optimal TED and the MAP based timing estimator.

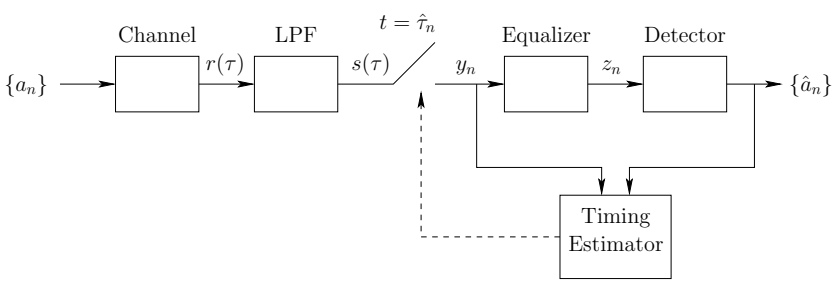

Fig. 2. Proposed timing recovery architecture.

\section{A. Proposed Timing Error Detector}

We first derive a timing error detector that is optimal in the sense that it provides sufficient statistics about the instantaneous timing error. Let $s^{\circ}(t)$ denote the ideal noisefree readback waveform:

$$
s^{\circ}(t)=\sum_{m} b_{m} h_{T}(t-m T)=\sum_{m} a_{m} h_{D}(t-m T)
$$

where $h_{D}(t)=\left(h_{T}(t)-h_{T}(t-T)\right) / 2$ is the dibit response. Denote its samples at $t=n T$ by

$$
y_{n}^{\circ}=s^{\circ}(n)=\sum_{m} a_{n-m} h_{D}(m T) .
$$

The superscript "o" indicates that the readback signal is ideal and devoid of imperfections due to clock jitter or noise.

Suppose that we have an estimate $\hat{\tau}_{n}$ of the sampling time $\tau_{n}$ which is obtained from an earlier step of the timing recovery algorithm. The corresponding sample of the filtered readback waveform can be written in terms of the ideal readback waveform using (3) as $y_{n}=s^{\circ}\left(f\left(\hat{\tau}_{n}\right)\right)+w\left(\hat{\tau}_{n}\right)$. Using the Taylor series expansion and the fact that the slope of $f(\cdot)$ is close to unity, we can approximate $y_{n}$ as follows:

$$
\begin{aligned}
y_{n} & =y_{n}^{\circ}+\left(\hat{\tau}_{n}-\tau_{n}\right)\left[\frac{d s^{\circ}(t)}{d t}\right]_{n T}+w\left(\hat{\tau}_{n}\right) \\
& =y_{n}^{\circ}+\gamma_{n}\left(\tau_{n}-\hat{\tau}_{n}\right)+w_{n}
\end{aligned}
$$

where

$$
\gamma_{n}:=-\left[\frac{d s^{\circ}(t)}{d t}\right]_{n T}=-\sum_{m} a_{n-m}\left[\frac{d h_{D}(t)}{d t}\right]_{m T}
$$

and $w_{n}=w\left(\hat{\tau}_{n}\right)$ is approximately IID with variance $\sigma_{W}^{2}=$ $N_{0} / 2 T$. Since $\hat{\tau}_{n}$ is a known quantity from a previous estimation step we can calculate the following quantity

$$
\theta_{n}:=\left(y_{n}-y_{n}^{\circ}\right)+\gamma_{n} \hat{\tau}_{n}=\gamma_{n} \tau_{n}+w_{n}
$$

which we term as the observation of $\tau_{n}$ at time $n$. Clearly $\theta_{n}$ contains the same information as $y_{n}$ and can be used as a sufficient statistic in our estimation. This is unlike the MM TED which is based on a heuristics argument. Note that we have assumed knowledge of the data bits $\left\{a_{n}\right\}$ to calculate $y_{n}^{\circ}$ and $\gamma_{n}$. In practice they can be estimated using the detected bits with a small delay. This issue is discussed in Section IV. For now let us assume that $\gamma_{n}$ and $y_{n}^{\circ}$ are known. 


\section{B. MAP Timing Estimation}

We showed in (12) that the observation at time $n$ is expressible as $\theta_{n}=B_{n} \lambda_{n}+w_{n}$, where

$$
B_{n}=\left(\gamma_{n}, 0\right)
$$

and $w_{n}$ is an IID Gaussian noise with variance $\sigma_{W}^{2}$ and $\lambda_{n}$ is described by the random walk model (4): $\lambda_{n}=A \lambda_{n-1}+v_{n}$. The timing estimator computes the optimal estimate of $\tau_{n+1}$ from the statistically sufficient observations $\left\{\theta_{k}: k \leq n\right\}$. Let the estimates and error covariances of $\lambda_{m}, m \geq n$ given $\theta_{0}^{n}:=\left\{\theta_{k}: k \leq n\right\}$ be denoted by

$$
\begin{aligned}
\hat{\lambda}_{m}^{(n)} & =\mathrm{E}\left[\lambda_{m} \mid \theta_{0}^{n}\right], \quad m \geq n \\
K_{m}^{(n)} & =\operatorname{cov}\left(\lambda_{m}-\hat{\lambda}_{m}^{(n)}\right) .
\end{aligned}
$$

Strictly speaking this is the MMSE estimate of the timing error. However, in this case the above estimates coincide with the MAP estimates because our measurements and noise are jointly Gaussian. The above problem can be solved using tools from Kalman filtering theory, the details of which are omitted here. The final result can be stated succinctly as a set of recursive update equations:

$$
\begin{aligned}
\hat{\lambda}_{n}^{(n)} & =\left(D_{n}^{T} C_{n}^{-1} D_{n}\right)^{-1} D_{n}^{T} C_{n}^{-1}\left(\begin{array}{c}
\theta_{n} \\
\hat{\lambda}_{n-1}^{(n-1)}
\end{array}\right) \\
K_{n}^{(n)} & =\left(D_{n}^{T} C_{n}^{-1} D_{n}\right)^{-1}
\end{aligned}
$$

where

$$
\begin{aligned}
C_{n} & =\left(\begin{array}{cc}
\sigma_{W}^{2} & 0 \\
0 & K_{n-1}+A^{-1} K_{v}\left(A^{-1}\right)^{T}
\end{array}\right) \\
D_{n} & =\left(\begin{array}{c}
B_{n} \\
A^{-1}
\end{array}\right) .
\end{aligned}
$$

Eq. (4) and the fact that $\left\{v_{k}: k>n\right\}$ is zero-mean and independent of $\theta_{0}^{n}$ imply that $\hat{\lambda}_{m}^{(n)}=A^{m-n} \hat{\lambda}_{n}^{(n)}$. Thus, estimates of all $\lambda_{m}$ for $m \geq n$ are easily calculated from $\lambda_{n}^{(n)}$.

The above estimation is similar in flavor to Kalman filtering based algorithms [4], [5], [7]. These algorithms assume the availability of measurements of the form $\theta_{n}=\tau_{n}+w_{n}$ without providing a specific structure for the TED itself. In contrast our TED (12), which provides sufficient statistics of the timing information, assumes the form $\theta_{n}=\gamma_{n} \tau_{n}+w_{n}$ where $\gamma_{n}$ is a (computable) data dependent gain. The derivation of the optimal TED is the novel aspect of our work.

\section{DECISION-DiRECTED IMPLEMENTATION}

Recall that $\gamma_{n}$ and $y_{n}^{\circ}$ need to be estimated from the decision bits. However they depend on future input bits. A workaround is to truncate the tail parts of the summations (8) and (11) and replace the actual bits by (delayed) detected bits, i.e.,

$$
\begin{aligned}
y_{n}^{\circ} & \simeq \sum_{m=-J}^{J_{0}} \hat{a}_{n-m} h_{D}(m T) \\
\gamma_{n} & \simeq-\sum_{m=-J}^{J_{0}} \hat{a}_{n-m}\left[\frac{d h_{D}(t)}{d t}\right]_{t=m T}
\end{aligned}
$$

where $J_{0}$ and $J$ are sufficiently large integers. In general, larger values of $J_{0}$ and $J$ yield better approximations. However, the above expression depends on $\hat{a}_{n+J}$ which implies an unavoidable delay of $J$ bits between the detector and the timing estimator. Although $J_{0}$ does not affect the loop delay, we can also choose a small value for $J_{0}$ for reduced complexity. If the equalizer has $K$ non-causal taps, this introduces a delay of $K$ samples in the forward link. Additionally, the detector introduces a certain delay. For instance the Viterbi detector, although a sequence detector, can be made to produce suboptimal decisions after decoding through a depth of $D$ bits in the trellis. This causes a delay of $D$ bits. Thus the total delay in the timing loop is $L=J+K+D$ and the implemented algorithm calculates $\lambda_{n+L+1}^{(n)}=A^{L+1} \lambda_{n}^{(n)}$. Finally, we point out that the transition response $h_{T}(t)$ used to compute $y_{n}^{\circ}$ and $\gamma_{n}$ can be estimated from a large training sequence.

Apart from its optimality, the MAP/MMSE estimation method is easier to design than the conventional MM method. For example, we need to specify only the initial covariance matrix $K_{0}^{(0)}$, while the MM method requires tuning of the loop gain parameters by trial and error. In typical systems, the initial phase offset is generally within one-half of a cycle and the frequency offset is less than $1 \%$. Thus, we can initialize the recursion with

$$
K_{0}^{(0)}=\left(\begin{array}{cc}
T^{2} / 4 & 0 \\
0 & 10^{-4}
\end{array}\right) .
$$

\section{A. Complexity Analysis}

The quantities (18) and (19) are both convolutions of two sequences where one of them is binary and the other is constant. Thus, rather than using arithmetic operations, we can implement these convolutions with a look-up table with $2^{m}$ entries where $m$ is the number of terms in the summation. Likewise, the convolution of the target filter with the data bits in the conventional algorithm can be implemented with a lookup table. Assume that the convolutions are implemented with look-up tables due to their negligible associated cost compared to arithmetic operations. For brevity we omit the details of how to optimally pipeline the computations and look-ups. Our complexity analysis reveals that the MM algorithm requires 4 multiplications and 4 additions per clock cycle (excluding equalization, detection and table look-ups). Whereas, our proposed algorithm including the Kalman filter requires 11 multiplications, 14 additions and 1 division per clock cycle, making it roughly four times computationally more complex than the conventional algorithm.

\section{Performance Results}

In this section, we evaluate the performance of the proposed timing recovery algorithm (Fig. 2) for an uncoded perpendicular magnetic recording channel at normalized recording density of $W / T=2$ by computer simulation and compare the results with those of a conventional method (Fig. 1) using the MM timing error detector based on the equalized sequence $z_{n}$. The MM loop filter parameters are chosen to minimize the convergence time for a constant frequency offset. This is done 
by placing the closed loop poles of the linearized feedback system (in the $Z$-domain) as close to the origin as possible. We process sectors of 4096 information bits and an additional 100 bits for the preamble. We use an origin-centered equalizer of length $2 K+1=11$ designed for a monic generalized partial response (GPR) target [9] of length 3, and a Viterbi detector with detection depth of $D=4$ bits. In the implementations of (8) and (11) we keep only $J=4$ non-causal terms and $J_{0}=10$ causal terms. Therefore, the net delay in the timing recovery loop is $L=K+D+J=13$ bits. We adopt the signal-to-noise ratio (SNR) definition SNR $:=E_{i} / N_{0}$ where $E_{i}$ is the energy in the derivative of the transition response $h_{T}(t)$ scaled by $W^{2}$ to preserve the units of energy [8]

$$
E_{i}=W^{2} \int_{\mathbb{R}}\left|h_{T}^{\prime}(t)\right|^{2} d t .
$$

The actual frequency offset encountered in a real system is the range of $0.2 \%$ to $0.4 \%$ and the initial phase offset is about 0.5 of a clock pulse. In all the simulations we choose an initial phase offset of $\tau_{0}=0.5 \mathrm{~T}$ and a frequency offset of $0.4 \%$, i.e., $\mu_{0}=1.004$. We also allow the local frequency to change slowly according to the random walk model described by (4) with $\sigma_{R}=\sigma_{S}=10^{-5}$.

We consider SNR values in the range $16 \mathrm{~dB}$ to $25 \mathrm{~dB}$. At $16 \mathrm{~dB}$ the SNR is considered very low as it produces raw bit error rates well in excess of $10^{-2}$. We evaluate the performance of the proposed and the conventional timing recovery algorithms in terms of their cycle slip rate (CSR) and timing error variance. Fig. 3 shows the normalized steady state timing error variance which is defined as

$$
\mathrm{E}\left\|\tau_{n}-\hat{\tau}_{n}\right\|^{2} / T^{2}
$$

where $\mathrm{E}(\cdot)$ denotes the expectation over $n$ as well as the ensemble. These values are obtained by processing sectors that do not have cycle slips. Clearly, the MAP/MMSE method yields a better estimate of the timing phase and hence a lower error variance for each SNR. Fig. 4 shows that the cycle slip rates are lower for MAP/MMSE timing recovery.

\section{CONCLUSION}

We investigated the problem of timing recovery for magnetic recording at low signal to noise ratio. We proposed a new decision directed TED that, unlike the TED of Mueller and Müller, provides sufficient statistics about instantaneous timing error. Using random walk evolution models for the clock phase and frequency offsets, we derive expressions for the MAP/MMSE optimal timing information from the readback samples. We showed that the estimates can be implemented efficiently using a set of recursive update equations. Simulation results show that this method performs better than a conventional timing recovery scheme in terms of both steady state timing error variance and cycle slip rates. Our analysis shows that the proposed algorithm has about 4 times the computational complexity of the conventional algorithm.

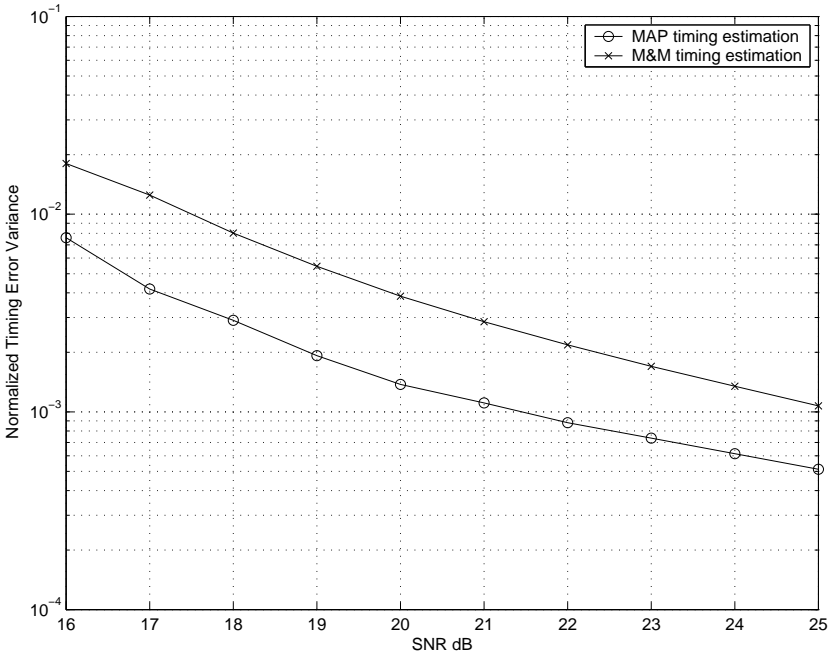

Fig. 3. Comparison of timing error variance performance.

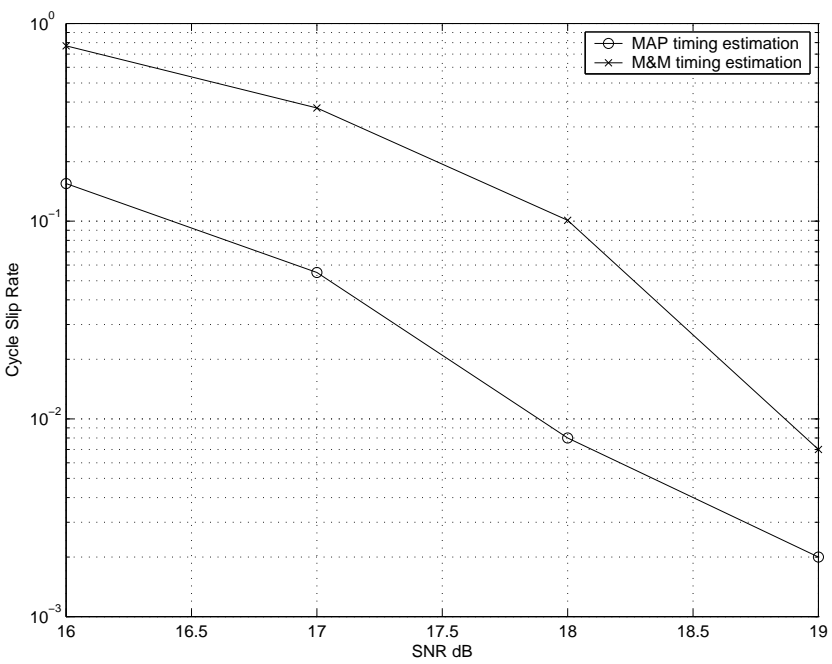

Fig. 4. Comparison of CSR performance.

\section{REFERENCES}

[1] K. Mueller and M. Müller, "Timing recovery in digital synchronous data receivers," IEEE Trans. on Comm., vol. COM-24, no. 5, pp. 516-531, May 1976.

[2] J. W. M. Bergmans, Digital Baseband transmission and recording, Kluwer Academic Publishers, 1996.

[3] H. Meys, M. Moeneclaey, and S. A. Fechtel, Digital Communication Receivers New York, USA, Wiley and Sons, 1998

[4] P. F. Driessen. "DPLL bit synchronizer with rapid acquisition using adaptive Kalman filtering techniques," IEEE Trans. on Comm., vol. 42, no. 9, pp. 2673-2675, September 1994.

[5] G. S. Christiansen, "Modeling of a PRML timing loop filter as a Kalman filter," GlobeCom '94, vol. 2., December 1994.

[6] T. M. Cover and J. A. Thomas, Elements of Information Theory, Wiley, New York, 1991

[7] J. Xie, L. Sun, and B. V. K. Vijaya Kumar, "Analysis of Kalman filter in timing acquisition in data storage read channels," IEEE Global Telecommunications Conf., vol. 4, pp. 23642368, Dec. 2005.

[8] Bane Vasic and Erozan Kurtas, Eds., Coding and Signal Processing for Magnetic Recording Systems, CRC Press, 2005.

[9] J. Moon and W. Zeng, "Equalization for maximum likelihood detectors," IEEE Trans. Magnetics, vol. 31, no. 2, pp. 1083-1088, March 1995. 